Vietnam Journal of Mechanics, VAST, Vol.42, No. 4 (2020), pp. 415-426

DOI: https://doi.org/10.15625/0866-7136/14765

\title{
LIMIT ANALYSIS OF MICROSTRUCTURES BASED ON HOMOGENIZATION THEORY AND THE ELEMENT-FREE GALERKIN METHOD
}

\author{
Phuc L. H. Ho ${ }^{1}$, Canh V. Le ${ }^{1, *}$ \\ ${ }^{1}$ Department of Civil Engineering, International University, VNU-HCMC, Vietnam \\ *E-mail: lvcanh@hcmiu.edu.vn
}

Received: 05 June 2020 / Published online: 23 December 2020

\begin{abstract}
This paper presents a novel numerical formulation of computational homogenization analysis of materials at limit state. The fluctuating displacement field are approximated using the Element-Free Galerkin (EFG) meshless method. The estimated yield surface of materials can be determined by handling the multiscale (macro-micro) transition. Taking advantage of high-order EFG shape function and the second-order cone programming, the resulting optimization problem can be solved rapidly with the great accuracy. Several benchmark examples will be investigated to demonstrate the computational efficiency of proposed method.
\end{abstract}

Keywords: homogenization, limit analysis, second-order cone programming, Element-Free Galerkin method.

\section{INTRODUCTION}

As the increasing use of composite and heterogeneous materials in practical engineering structures, the estimation of their effective properties plays a vital role in safety assessment as well as structural design. The elastic-plastic incremental method can be employed to predict the ultimate load and collapse mechanism of structures. However, direct method, e.g. limit analysis shows more effectively, i.e. the critical status of structures can be determined without any knowledge of whole loading path history [1,2].

Dealing with the microstructures, the multi-scale homogenization technique or socalled global-local analysis firstly introduced in [3] has been widely exploited in recent years. The computational homogenization methodology has been mostly applied to the periodic composite and heterogeneous materials. Based on the concept of representative volume element (RVE) and homogenization technique in [3], the first formulation of limit analysis in terms of solving the composite structure at micro-scale has been proposed in [4]. Then, the theoretical formulation was developed by [5,6] for fiber-reinforced composite using Drucker-Prager, Mohr-Coulomb or von Mises yield criterion. The first numerical implementation for this field has been reported in [7] with the use of finite 
element method and linear mathematical programming. By means of static direct methods, a three-dimensions finite element procedure for analysis of isotropic microstructures was developed by $[8,9]$. Besides, using the similar approach, a quasi-lower bound formulation for periodic composite and heterogeneous materials using the nonlinear programming was presented in [10]. In contrast, the kinematic formulations in combination with nonlinear algorithms can be found in [11-15]. In those works, both of isotropic and anisotropic materials obeying the von Mises or elliptic yield criterion were considered. In order to improve the computational aspect, a numerical procedure based on combination of kinematic limit analysis and homogenization theory for periodic materials proposed in [16]. However, these studies only focused on the application of finite element method.

This study aims to develop a novel computational homogenization approach for upper bound limit analysis of microstructures using Element-Free Galerkin method known as one of most successful procedures for yield design problems. The stability conforming nodal integration (SCNI) technique proposed in [17] is employed to improve the performance of the numerical formulation. In addition, the plastic dissipation will be transformed into the form of a sum of norms and the resulting optimization are then formulated as conic one. The benchmark numerical examples will be considered and the good agreement in comparison to previous procedures proves the performance of present method.

\section{LIMIT ANALYSIS BASED ON HOMOGENIZATION THEORY}

\subsection{Homogenization theory}

Consider a heterogeneous representative volume element (RVE) $\Omega \in \mathbb{R}^{2}$ at every material point $\mathbf{x} \in V$, where $V \in \mathbb{R}^{2}$ denotes the heterogeneous macroscopic-continuum. The micro-structure is subjected to the body force $\mathbf{f}$, the surface load $\mathbf{t}$ on the static boundary $\Gamma_{t}$ and fixed by the displacement field $\mathbf{u}$ on the kinematic boundary $\Gamma_{u}$.

The micro-scale problem can be treated as the boundary value one in solid mechanics, where the overall strain $\mathbf{E}$ are transferred to micro-structure in form of kinematic boundary constrains. At microscopic scale, the local fields is decomposed into two parts including mean and fluctuation terms. The displacement, strain and stress fields of microstructure are now given by

$$
\begin{aligned}
& \mathbf{u}(\mathbf{x})=\mathbf{E} \cdot \mathbf{X}+\tilde{\mathbf{u}}(\mathbf{x}), \\
& \boldsymbol{\epsilon}(\mathbf{x})=\mathbf{E}+\tilde{\boldsymbol{\epsilon}}(\mathbf{x}), \\
& \sigma(\mathbf{x})=\boldsymbol{\Sigma}+\tilde{\sigma}(\mathbf{x}),
\end{aligned}
$$

where matrix $\mathbf{X}$ consists of the position components of each material point in the computational domain; $\Sigma$ is the overall stress; $\tilde{\mathbf{u}}(\mathbf{x}), \tilde{\boldsymbol{\epsilon}}(\mathbf{x})$ and $\tilde{\boldsymbol{\sigma}}(\mathbf{x})$ denote the fluctuation parts of displacement, strain and stress rate.

For the enforcement of boundary conditions, the most efficient in terms of convergence rate so-called periodic procedure, where there are the periodicity of fluctuation displacement field and anti-periodicity of traction field on RVE boundary, is employed 
in this study

$$
\begin{aligned}
\tilde{\mathbf{u}}\left(\mathbf{x}^{+}\right) & =\tilde{\mathbf{u}}\left(\mathbf{x}^{-}\right), & & \text {on } \Gamma_{u} \\
\mathbf{t}\left(\mathbf{x}^{+}\right) & =-\mathbf{t}\left(\mathbf{x}^{-}\right), & & \text {on } \Gamma_{t}
\end{aligned}
$$

where $\tilde{\mathbf{u}}\left(\mathbf{x}^{+}\right)$and $\tilde{\mathbf{u}}\left(\mathbf{x}^{-}\right)$are the fluctuation displacement field, $\mathbf{t}\left(\mathbf{x}^{+}\right)$and $\mathbf{t}\left(\mathbf{x}^{-}\right)$are the traction field of positive and negative boundaries, respectively.

Note that regarding to the periodic characteristic of the fluctuation terms, the average of $\tilde{\boldsymbol{\epsilon}}(\mathbf{x})$ and $\tilde{\boldsymbol{\sigma}}(\mathbf{x})$ over the RVE should vanish, it means

$$
\langle\tilde{\boldsymbol{\epsilon}}\rangle=0, \quad\langle\tilde{\boldsymbol{\sigma}}\rangle=0,
$$

where the operation $\langle\cdot\rangle$ stands the volume average of fields over the RVE. Denoting $|\Omega|$ for the area of RVE, the macroscopic quantities can be calculated from the microscopic ones via the average relations

$$
\mathbf{E} \equiv\langle\boldsymbol{\epsilon}\rangle=\frac{1}{|\Omega|} \int_{\Omega} \epsilon \mathrm{d} \Omega, \quad \Sigma \equiv\langle\sigma\rangle=\frac{1}{|\Omega|} \int_{\Omega} \sigma \mathrm{d} \Omega
$$

For any admissible velocity and stress field satisfying the periodic and anti-periodic conditions on boundary, the principle of macroscopic virtual work can be expressed as

$$
\langle\sigma: \epsilon\rangle=\Sigma: \mathrm{E} .
$$

\subsection{Fundamental of limit analysis}

Denoting $\mathcal{X}$ and $\mathcal{Y}$ for the appropriate spaces of statically admissible stress state and of kinematically admissible velocity state, respectively. The exact collapse multiplier will be obtained if one of following optimization problems is solved

$$
\begin{aligned}
\lambda_{\text {exact }} & =\max \{\lambda \mid \exists \sigma \in \mathcal{B}: a(\sigma, \mathbf{u})=\lambda F(\mathbf{u}), \forall \mathbf{u} \in \mathcal{Y}\} \\
& =\max _{\sigma \in \mathcal{B}} \min _{\mathbf{u} \in \mathcal{C}} a(\sigma, \mathbf{u}) \\
& =\min _{\mathbf{u} \in \mathcal{C}} \max _{\sigma \in \mathcal{B}} a(\sigma, \mathbf{u}) \\
& =\min _{\mathbf{u} \in \mathcal{C}} D(\mathbf{u}),
\end{aligned}
$$

where $\mathcal{C}=\{\mathbf{u} \in \mathcal{Y} \mid F(\mathbf{u})=1\} ; \mathcal{B}=\{\sigma \in \mathcal{X} \mid \psi(\sigma) \leq 0\}$; the plastic dissipation rate $D(\mathbf{u})$ and the external work $F(\mathbf{u})$ can be expressed in terms of $\sigma$ and $\mathbf{u}$ as follow

$$
\begin{aligned}
D(\mathbf{u}) & =\max _{\sigma \in \mathbb{B}} a(\sigma, \mathbf{u}), \\
F(\mathbf{u}) & =\int_{\Omega} \mathbf{f}^{T} \mathbf{u} \mathrm{d} \Omega+\int_{\Gamma_{t}} \mathbf{t}^{T} \mathbf{u d} \Gamma .
\end{aligned}
$$

It is worth noting that most of yield criterion can be expressed in the following form

$$
\psi(\sigma)=\sqrt{\sigma^{T} \mathbf{P} \sigma}-1,
$$

where $\mathbf{P}$ is the coefficient matrix consisting of strength properties of materials. For isotropic materials, the von Mises criterion is frequently applied, and matrix $\mathbf{P}$ for plane 
stress problem can be expressed as

$$
\mathbf{P}=\frac{1}{\sigma_{p}}\left[\begin{array}{ccc}
1 & -1 / 2 & 0 \\
-1 / 2 & 1 & 0 \\
0 & 0 & 3
\end{array}\right],
$$

where $\sigma_{p}$ is the isotropic yield stress of material.

In framework of limit analysis, the strain rates are assumed to obey the normality rule. Denoting $\boldsymbol{\Theta}$ for the inversion matrix of $\mathbf{P}$, the power of dissipation can be formulated in terms of strain rates as

$$
D(\epsilon)=\int_{\Omega} \sqrt{\epsilon^{T} \Theta \epsilon} \mathrm{d} \Omega .
$$

\subsection{Kinematic limit analysis based on homogenization theory}

Assuming that all constitutions of ductile composite are rigid-perfectly plastic and the strain of constitutions obey the normality rule. The kinematic approach in framework of limit analysis for computation homogenization described in [11, 12, 14, 15, 18, 19] will be taken into account in this paper. According to the homogenization theory, all variables related to the microscopic structures are split into two parts including mean fields averaged over RVE and fluctuation fields.

Omitting the body force $\mathbf{f}$ and applying the principle of microscopic virtual work (5), the normalization condition of external power can be rewritten as

$$
F(\mathbf{u})=\int_{\Gamma_{t}} \mathbf{t}^{T} \mathbf{u d} \Gamma=\Sigma^{T} \mathbf{E}=1
$$

Now, the kinematic limit formulation of computational homogenization analysis for a periodic micro-structure can be expressed as

$$
\begin{array}{r}
\lambda^{+}=\min \int_{\Omega} \sqrt{\boldsymbol{\epsilon}^{T} \boldsymbol{\Theta} \epsilon} \mathrm{d} \Omega \\
\text { s.t }\left\{\begin{array}{l}
\Sigma^{T} \mathbf{E}=1 \\
\tilde{\mathbf{u}} \text { periodic on } \Gamma_{u}
\end{array}\right.
\end{array}
$$

Solving the nonlinear problem (12), the upper-bound of macroscopic limit strength $\lambda^{+} \Sigma$ will be determined. It should be noted that present study only considers the continuous velocity fields, and in cases when the velocity fields are assumed to be discontinuous, the dissipated power generated by discontinuities must be taken into account.

\section{ELEMENT-FREE GALERKIN METHOD}

The approximate function at point $\mathbf{x}$ reflected via a set of scattered nodes in arbitrary domain $\Omega$ using moving least square (MLS) technique can be expressed as

$$
u^{h}(\mathbf{x})=\sum_{i=1}^{m} p_{i}(\mathbf{x}) a_{i}(\mathbf{x})=\mathbf{p}^{T}(\mathbf{x}) \mathbf{a}(\mathbf{x}),
$$


where the coefficient vector $\mathbf{a}(\mathbf{x})$ and the basis function vector $\mathbf{p}(\mathbf{x})$ are defined by

$$
\begin{aligned}
\mathbf{a}(\mathbf{x}) & =\left[a_{1}(\mathbf{x}), a_{2}(\mathbf{x}), \ldots, a_{m}(\mathbf{x})\right], \\
\mathbf{p}^{T}(\mathbf{x}) & =\left[p_{1}(\mathbf{x}), p_{2}(\mathbf{x}), \ldots, p_{m}(\mathbf{x})\right] .
\end{aligned}
$$

The complete polynomial basis is given by

$$
\mathbf{p}^{T}(\mathbf{x})=\left[1, x, y, x^{2}, x y, y^{2}\right]
$$

The unknown coefficient $a_{i}(\mathbf{x})$ is a function of $\mathbf{x}, \mathbf{a}(\mathbf{x})$ is determined such that the weighted, discrete $L_{2}$ norm

$$
J(\mathbf{x})=\sum_{i=1}^{\mathcal{N}} w\left(\mathbf{x}-\mathbf{x}_{i}\right)\left[\mathbf{p}^{T}(\mathbf{x}) \mathbf{a}(\mathbf{x})-u_{i}\right],
$$

is minimized, where $u_{i}$ denotes the nodal value of approximate function at point $i^{\text {th }}, \mathcal{N}$ is number of points in the neighborhood of $\mathbf{x}, w\left(\mathbf{x}-\mathbf{x}_{i}\right)$ is the weighting function depending on the influent domain of point $\mathbf{x}$. This study employs the isotropic quadratic spline

$$
w\left(\mathbf{x}-\mathbf{x}_{i}\right) \equiv w_{i}(\mathbf{x})= \begin{cases}1-6 s_{i}^{2}+8 s_{i}^{3}-3 s_{i}^{4}, & \text { if } s_{i} \leq 1 \\ 0, & \text { if } s_{i} \leq 1\end{cases}
$$

where $s_{i}=\frac{\left\|\mathbf{x}-\mathbf{x}_{i}\right\|}{R_{i}}$, with $R_{i}$ is the support radius of node $i^{t h}$.

Minimizing $J(\mathbf{x})$ in Eq. (16) leads to the system linear equation

$$
\mathbf{A}(\mathbf{x}) \mathbf{a}(\mathbf{x})=\mathbf{B}(\mathbf{x}) \mathbf{u}
$$

with

$$
\begin{aligned}
\mathbf{u} & =\left[u_{1}, u_{2}, \ldots, u_{\mathcal{N}}\right] \\
\mathbf{A}(\mathbf{x}) & =\sum_{I=1}^{\mathcal{N}} w\left(\mathbf{x}-\mathbf{x}_{I}\right) \mathbf{p}\left(\mathbf{x}_{I}\right) \mathbf{p}^{T}\left(\mathbf{x}_{I}\right) \\
\mathbf{B}(\mathbf{x}) & =\left[w\left(\mathbf{x}-\mathbf{x}_{1}\right) p\left(\mathbf{x}_{1}\right), w\left(\mathbf{x}-\mathbf{x}_{2}\right) p\left(\mathbf{x}_{2}\right), \ldots, w\left(\mathbf{x}-\mathbf{x}_{\mathcal{N}}\right) p\left(\mathbf{x}_{\mathcal{N}}\right)\right] .
\end{aligned}
$$

Taking an inversion, $a(\mathbf{x})$ can be obtained by solving Eq. (18) as

$$
\mathbf{a}(\mathbf{x})=\mathbf{A}^{-1}(\mathbf{x}) \mathbf{B}_{I}(\mathbf{x}) \mathbf{u} \text {. }
$$

Now, the approximate function can be expressed as

$$
u^{h}(\mathbf{x})=\sum_{i=1}^{\mathcal{N}} \boldsymbol{\Phi}_{i}(\mathbf{x}) u_{i}
$$

where the EFG shape function is defined by

$$
\boldsymbol{\Phi}_{i}(\mathbf{x})=\mathbf{p}^{T}(\mathbf{x}) \mathbf{A}^{-1}\left(\mathbf{x}_{i}\right) \mathbf{B}_{i}(\mathbf{x})
$$

In order to improve the computational efficiency of the EFG method, the stabilized conforming nodal integration presented in [17] is applied here to obtain stabilized shape function derivatives as

$$
\tilde{\boldsymbol{\Phi}}_{i, \alpha}=\frac{1}{A_{C}} \oint_{\Gamma_{C}} \boldsymbol{\Phi}_{i}(\mathbf{x}) \mathbf{n}_{\alpha}(\mathbf{x}) \mathrm{d} \Gamma
$$


where $A_{C}$ is the area of a Voronoi smoothing domain with boundary $\Gamma_{C}, \mathbf{n}_{\alpha}$ is the outward surface normal vector. Detailed calculation of the smoothing shape function derivatives $\tilde{\boldsymbol{\Phi}}_{i, \alpha}$ can be found in [19].

\section{DISCRETE FORMULATION USING EFG METHOD}

In the kinematic formulation, the local microscopic fluctuation displacement $\tilde{u}(\mathbf{x})$ and fluctuation strain $\tilde{\boldsymbol{\epsilon}}(\mathbf{x})$ at point $\mathbf{x}$ can be approximated using EFG method as

$$
\begin{aligned}
\tilde{u}^{h}(\mathbf{x}) & =\sum_{i=1}^{\mathcal{N}} \boldsymbol{\Phi}_{i}(\mathbf{x}) \tilde{u}_{i}=\mathbf{N}(\mathbf{x}) \mathbf{d}, \\
\tilde{\boldsymbol{\epsilon}}(\mathbf{x}) & =\sum_{i=1}^{\mathcal{N}} \tilde{\boldsymbol{\Phi}}_{i, \alpha}(\mathbf{x}) \tilde{u}_{i}=\mathbf{B}(\mathbf{x}) \mathbf{d},
\end{aligned}
$$

where $\mathbf{N}(\mathbf{x})$ denotes the $\mathrm{iRBF}$ shape function; $\mathbf{B}(\mathbf{x})$ is the strain-displacement matrix consisting the smoothed version of shape function derivatives; and $\mathbf{d}$ is the vector containing the nodal fluctuation displacement components.

With the use of SCNI technique for the numerical integration, the plastic dissipation well-known as the objective function of the optimization problems can be expressed as

$$
D_{p}(\boldsymbol{\epsilon})=\sum_{i=1}^{\mathcal{N}} \sigma_{p} A_{i} \sqrt{(\mathbf{E}+\mathbf{B d})^{T} \mathbf{\Theta}(\mathbf{E}+\mathbf{B d})},
$$

where $\sigma_{p}$ is the yield stress of material, $A_{i}$ is the area of the $i^{\text {th }}$ nodal representative domain created using Voronoi diagrams.

In this study, the optimization problem will be formulated in the form of second order cone programming (SOCP) ensuring that it can be solved using the highly efficient solves. Hence, a form of sum of norm can be used to calculate the internal dissipation power as

$$
D_{p}(\boldsymbol{\epsilon})=\sum_{i=1}^{N} \sigma_{p} A_{i}\left\|\boldsymbol{\rho}_{i}\right\|
$$

where $\|\cdot\|$ denotes the Euclidean norms and $\sigma$ is the vector of additional variables defined by

$$
\boldsymbol{\rho}_{i}=\mathbf{Q}^{T}(\mathbf{E}+\mathbf{B d}),
$$

with $\mathbf{Q}$ denotes the Cholesky factor of $\boldsymbol{\Theta}$.

Next, the periodic feature of the fluctuation displacement for nodes on the boundary of RVE need to be enforced. Denoting $\Gamma^{+}$and $\Gamma^{-}$for the positive and negative boundary such that $\Gamma^{+} \cup \Gamma^{-}=\Gamma$ and $\Gamma^{+} \cap \Gamma^{-}=\varnothing$, the periodic boundary condition for each pair $\left\{\mathbf{x}^{+}, \mathbf{x}^{-}\right\}$of boundary material points can be expressed as

$$
\tilde{\mathbf{u}}\left(\mathbf{x}^{+}\right)-\tilde{\mathbf{u}}\left(\mathbf{x}^{-}\right)=0 .
$$

Assembling to the global matrix, Eq. (28) can be rewritten as

$$
\mathrm{Cd}=0 \text {. }
$$


Finally, by introducing the auxiliary variables $\left(t_{1}, t_{2}, \ldots, t_{\mathcal{N}}\right)$, the optimization problem can be formulated in the form of conic programming as follows

$$
\begin{aligned}
\lambda^{+}=\min & \sum_{i=1}^{N} \sigma_{p} A_{i}\left\|\boldsymbol{\rho}_{i}\right\| \\
\text { s.t } & \left\{\begin{array}{l}
\Sigma^{T} \mathbf{E}=1 \\
\mathbf{C d}=0 \\
\left\|\boldsymbol{\rho}_{i}\right\| \leq t_{i}, \quad i=1,2, \ldots, \mathcal{N}
\end{array}\right.
\end{aligned}
$$

\section{NUMERICAL EXAMPLES}

In this section, the computational aspect of proposed method will be investigated. An unit RVE of $a \times a=1 \times 1 \mathrm{~mm}$ is used for all examples. The problems are considered in plane stress, and thus number of variables $\mathcal{N}_{\text {var }}$ is equal to $6 \times \mathcal{N}+3$. The resultant optimization problems are solved using the commercial software package Mosek on a 2.8 GHz Intel Core i7 PC running Window 10.

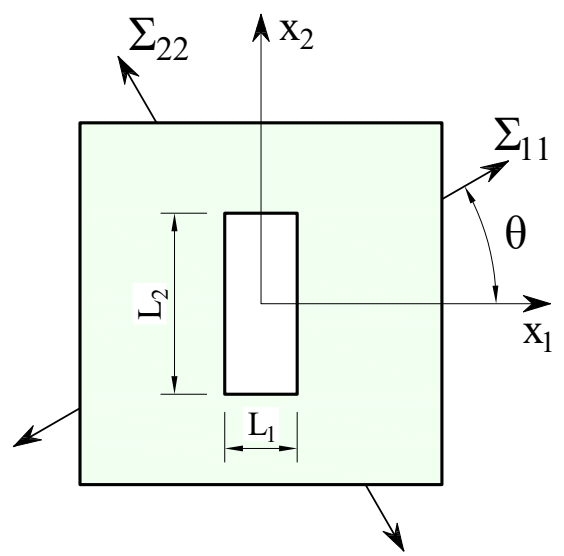

(a) Rectangular hole RVE

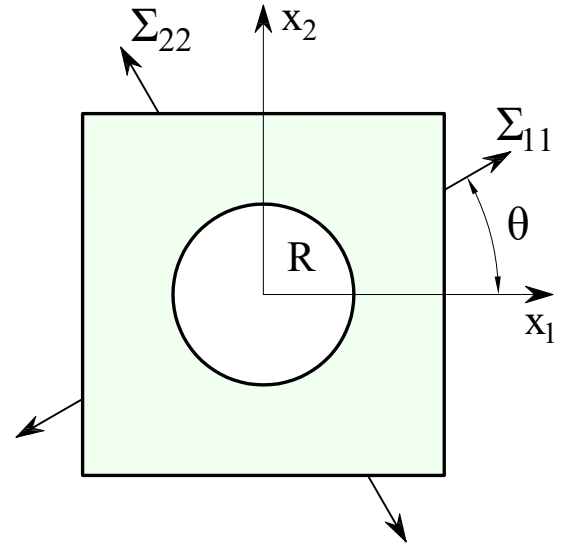

(b) Circular hole RVE

Fig. 1. RVEs of perforated materials: geometry, loading and dimension

The effect of microscopic hole on the overall strength of perforated materials is investigated. Two perforated material models including a rectangular hole of size $L_{1} \times L_{2}$ and a circle hole of radius $R$ at center are considered. The problems have been investigated using kinematic formulation in $[11,12,16]$ and quasi-static formulation in [10]. The RVE is subjected to an orthogonal macroscopic stress $\left(\Sigma_{11}, \Sigma_{22}\right)$ in plane $\left(x_{1}, x_{2}\right)$ as shown in Fig. 1, where $\theta$ denotes the angle between the principle stress and $x_{1}$-axis. The matrix material of rectangular hole RVE is aluminum Al whose yield stress is $\sigma_{p}=137 \mathrm{MPa}$, while those of RVE with circular hole is mild steel St3s with yield stress $\sigma_{p}=273 \mathrm{MPa}$. Material behavior is assumed to obey the von Mises yield criterion. Fig. 2 illustrates the scheme of nodal discretization using Voronoi diagram. 


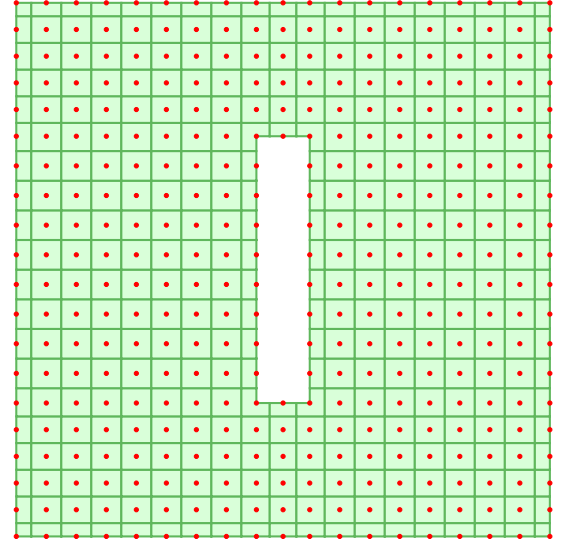

(a) Rectangular hole RVE $\left(L_{1} \times L_{2}=\right.$ $0.1 \times 0.5 \mathrm{~mm})$

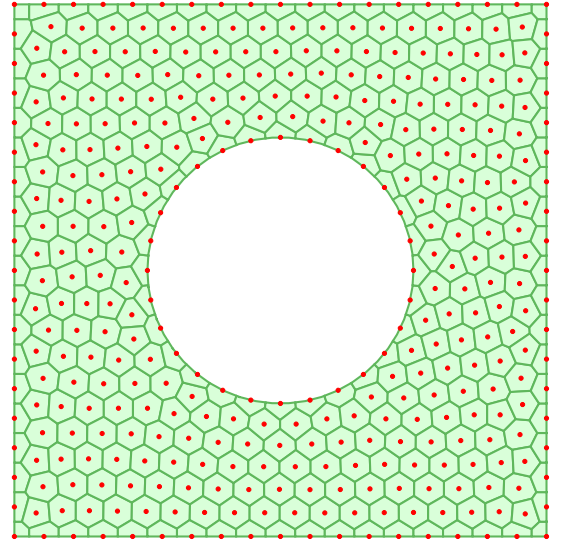

(b) Circular hole $\operatorname{RVE}(R=0.25 \times a)$

Fig. 2. RVEs of perforated materials: nodal discretization using Voronoi cells

Table 1. Rectangular hole RVE $\left(L_{1} \times L_{2}=0.1 \times 0.5 \mathrm{~mm}, \theta=0^{\circ}\right)$

\begin{tabular}{cccc}
\hline Author and approach & $\Sigma_{11} / \sigma_{p}$ & sdof & CPU-Time (s) \\
\hline Present study, EFG & 0.5559 & 6228 & 17 \\
Li et al. [12], FEM & 0.5600 & 1920 & 95 \\
Le et al. [16], FEM & 0.5561 & 8140 & 6 \\
\hline
\end{tabular}

sdof denotes the total system degrees of freedom

In case of rectangular hole RVE, the problem is considered with different dimensions of hole involving $\left(L_{1} \times L_{2}=0.1 \times 0.5 \mathrm{~mm}\right)$ and $\left(L_{1} \times L_{2}=0.1 \times 0.7 \mathrm{~mm}\right)$. Tab. 1 shows present numerical solutions compared with those in $[12,16]$. It can be observed that proposed procedure can prove the highly accurate solutions with low computational cost in comparison with approaches presented in $[12,16]$. The number of variables in present procedure is less than those used in [16], while the numerical result is approximate. Moreover, with the use of cone-based algorithm the resultant optimization problem in present study can be solved rapidly, the CPU-time required for handling the problem is much less than those in [12] using iterative algorithm. Fig. 3 plots the macroscopic uniaxial strength $\Sigma_{11}$ for various values of angle $\theta$ corresponding to two different sizes of rectangular hole. It can be seen from both of sub-figures that the upper-bound solutions obtained using present iRBF are slightly lower (better) than available those in other studies. The figure also shows the good agreement of present solutions in comparison with previous numerical results reported in $[12,16]$ as well as the experimental results reported by Litewka et al. (ref [12]).

For the case of perforated material with circular hole, various values of ratio $R / a$ and loading angle $\theta$ are considered. Fig. 4 also plots the solutions reported in $[11,16]$. 


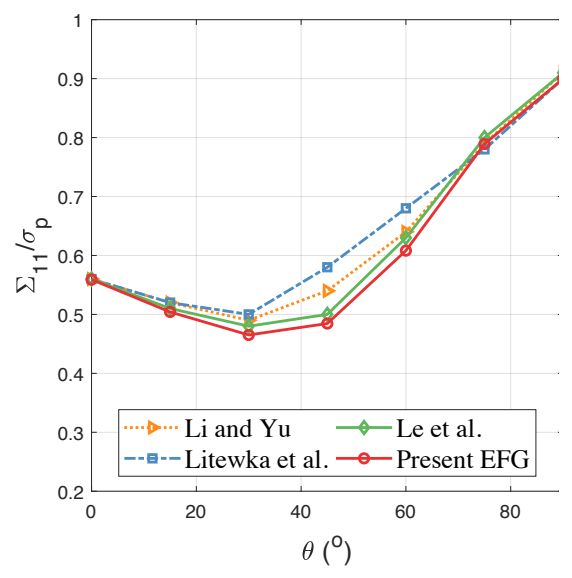

(a) $L_{1} \times L_{2}=0.1 \times 0.5 \mathrm{~mm}$

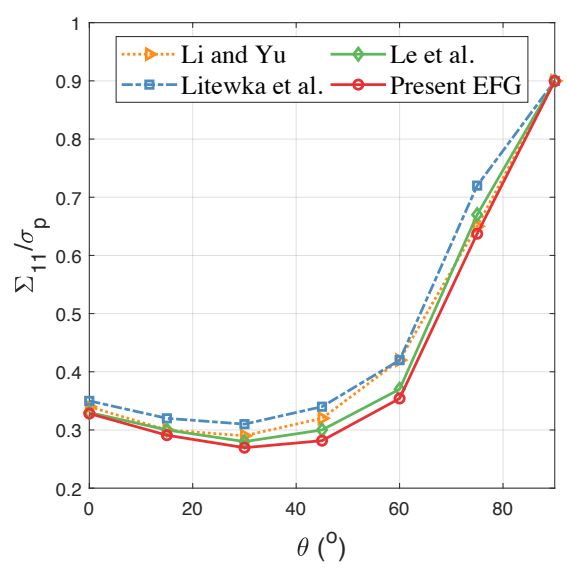

(b) $L_{1} \times L_{2}=0.1 \times 0.7 \mathrm{~mm}$

Fig. 3. Rectangular hole RVE: limit uniaxial strength $\Sigma_{11}$ in comparison with other procedures

From the comparison in the Figure, it can be observed that present solutions are in good agreement in comparison with available those in other numerical methods.

Next, the effect of microscopic hole size on the overall strength of perforated materials is investigated. Obviously, it is reasonable that the effective macroscopic strength decreases when increasing size of holes. For rectangular hole RVE, it can be observed that for all values of angle $\theta$, the obtained macroscopic strengths in case of $L_{1} \times L_{2}=$ $0.1 \times 0.7 \mathrm{~mm}$ are lower than those in case of $L_{1} \times L_{2}=0.1 \times 0.5 \mathrm{~mm}$, as seen in Fig. 3. For the case of RVE with circular hole, the solutions in Fig. 4 shows the similar trend when increasing ratio $R / a$.

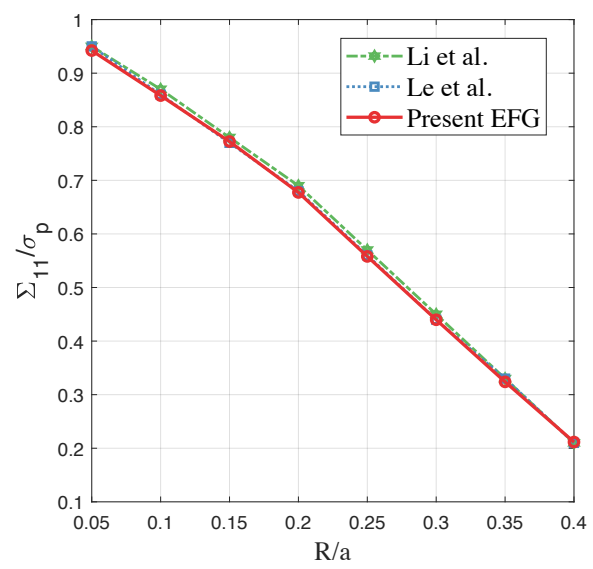

(a) $\theta=0^{\circ}$

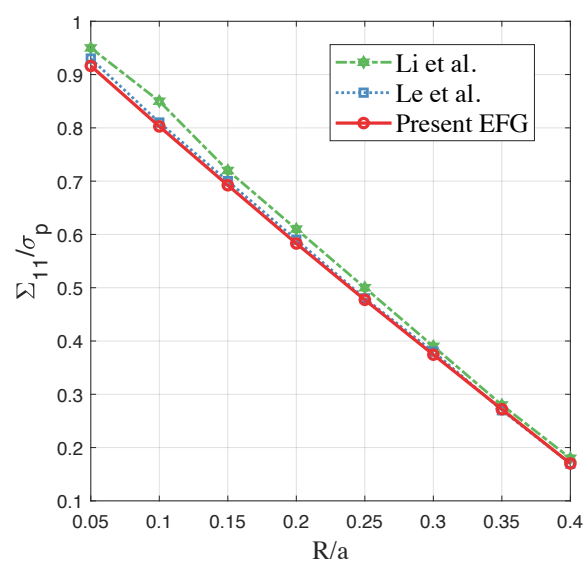

(b) $\theta=45^{\circ}$

Fig. 4. Circular hole RVE: limit uniaxial strength $\Sigma_{11}$ in comparison with other procedures 
The approximate macroscopic strength domain of perforated materials with rectangular hole $\left(L_{1} \times L_{2}=0.1 \times 0.5 \mathrm{~mm}\right)$ and circular hole $(R=0.25 \times a)$ for $\theta=0^{\circ}$ and $\theta=45^{\circ}$ are illustrated in Fig. 5. The strength in $\Sigma_{22}$ direction is greater than that in $\Sigma_{11}$ direction in case of rectangular hole RVE, while those are equivalent in case of circular hole RVE. The plastic dissipation distributions representing the failure mechanisms of RVEs are also presented in Figs. 6 and 7.

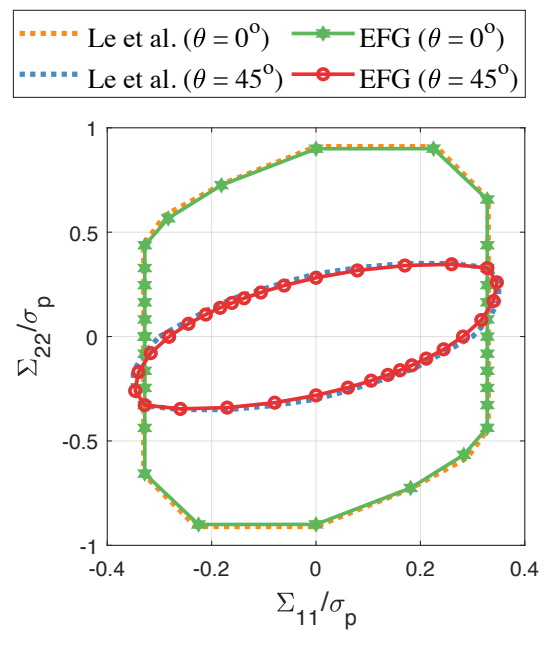

(a) Rectangular hole RVE

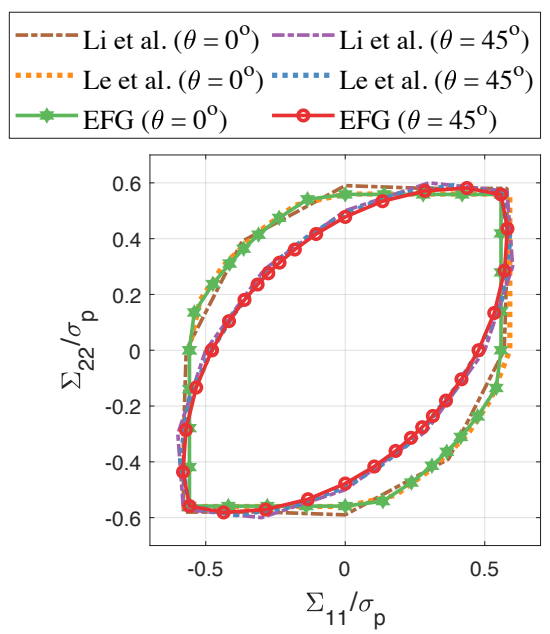

(b) Circular hole RVE

Fig. 5. Perforated materials: macroscopic strength domain at limit state

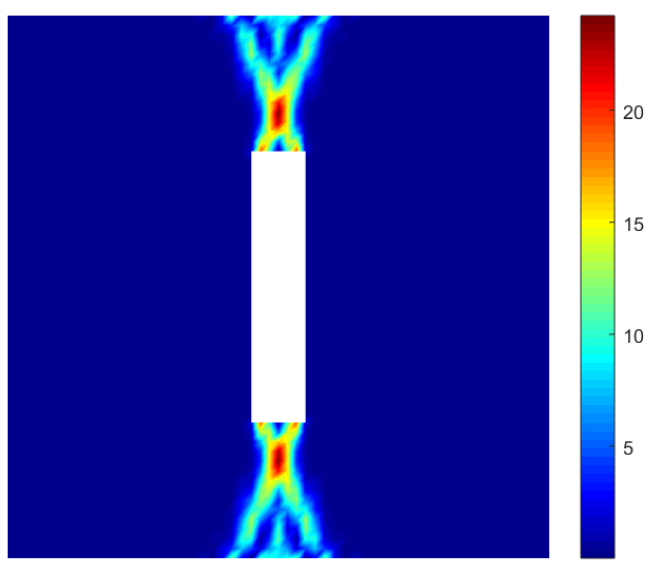

(a) $\theta=0^{\circ}$

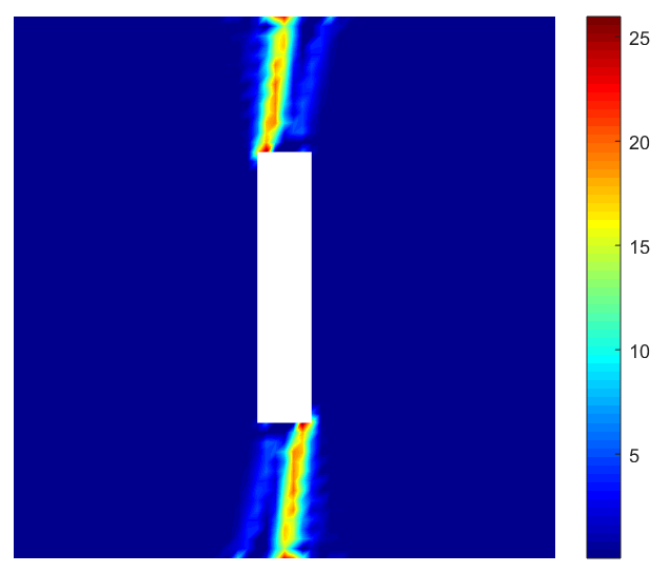

(b) $\theta=45^{\circ}$

Fig. 6. Rectangular hole RVE $\left(L_{1} \times L_{2}=0.1 \times 0.5 \mathrm{~mm}\right)$ : the distribution of plastic dissipation 


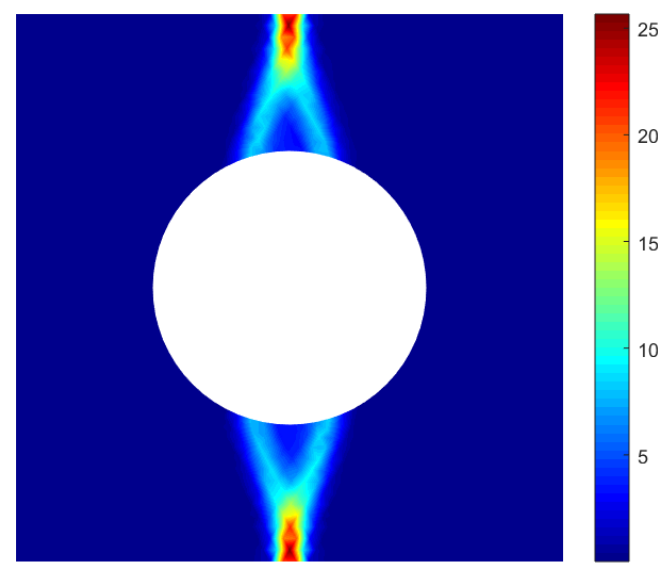

(a) $\theta=0^{\circ}$

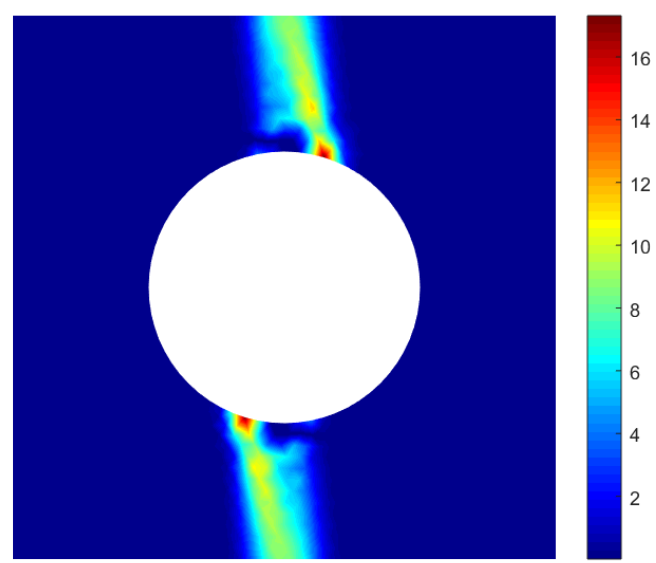

(b) $\theta=45^{\circ}$

Fig. 7. Circular hole RVE $(R=0.25 \times a)$ : the distribution of plastic dissipation

\section{CONCLUSIONS}

The plastic limit strength and the collapse mechanism of materials has been studied using the combination of direct analysis and homogenization theory. By means of second-order cone programming and the EFG approximation, the resulting optimization problems are kept in minimum size and solved rapidly. The good agreement of numerical solutions in comparison with other studies shows the computational efficiency of proposed method. In future work, the plane strain or three-dimensions problems, for which the volumetric locking phenomena is required to be handled, are extended. In addition, more complicate effects, e.g. material interfaces, multiple crack or even variable loading should be considered.

\section{ACKNOWLEDGMENT}

This research is funded by Vietnam National Foundation for Science and Technology Development (NAFOSTED) under grant number 107.02-2018.31.

\section{REFERENCES}

[1] M. A. Save, C. E. Massonnet, and C. C. Massonnet. Plastic analysis and design of plates, shells and disks, Vol. 15. North-Holland, (1972).

[2] J. Salençon. Yield design. John Wiley \& Sons, (2013).

[3] P. M. Squet. Local and global aspects in the mathematical theory of plasticity. Plasticity Today, (1985), pp. 279-309.

[4] D. E. Buhan. A homogenization approach to the yield strength of composite materials. European Journal of Mechanics, A/Solids, 10, (2), (1991), pp. 129-154.

[5] A. Taliercio. Lower and upper bounds to the macroscopic strength domain of a fiberreinforced composite material. International Journal of Plasticity, 8, (6), (1992), pp. 741-762. https://doi.org/10.1016/0749-6419(92)90026-9. 
[6] A. Taliercio and P. Sagramoso. Uniaxial strength of polymeric-matrix fibrous composites predicted through a homogenization approach. International Journal of Solids and Structures, 32, (14), (1995), pp. 2095-2123. https://doi.org/10.1016/0020-7683(94)00139-n.

[7] P. Francescato and J. Pastor. Lower and upper numerical bounds to the off-axis strength of unidirectional fiber-reinforced composites by limit analysis methods. European Journal of Mechanics-A/Solids, 16, (2), (1997), pp. 213-234.

[8] D. Weichert, A. Hachemi, and F. Schwabe. Application of shakedown analysis to the plastic design of composites. Archive of Applied Mechanics, 69, (9-10), (1999), pp. 623-633. https://doi.org/10.1007/s004190050247.

[9] D. Weichert, A. Hachemi, and F. Schwabe. Shakedown analysis of composites. Mechanics Research Communications, 26, (1999), pp. 309-18. https://doi.org/10.1016/s0093-6413(99)000294.

[10] H. Zhang, Y. Liu, and B. Xu. Plastic limit analysis of ductile composite structures from micro-to macro-mechanical analysis. Acta Mechanica Solida Sinica, 22, (1), (2009), pp. 73-84. https://doi.org/10.1016/s0894-9166(09)60092-6.

[11] H. Li, Y. Liu, X. Feng, and Z. Cen. Limit analysis of ductile composites based on homogenization theory. Proceedings of the Royal Society of London. Series A: Mathematical, Physical and Engineering Sciences, 459, (2031), (2003), pp. 659-675. https://doi.org/10.1098/rspa.2002.1039.

[12] H. X. Li and H. S. Yu. Limit analysis of composite materials based on an ellipsoid yield criterion. International journal of plasticity, 22, (10), (2006), pp. 1962-1987. https://doi.org/10.1016/j.ijplas.2006.02.001.

[13] H. X. Li. Limit analysis of composite materials with anisotropic microstructures: A homogenization approach. Mechanics of Materials, 43, (10), (2011), pp. 574-585. https://doi.org/10.1016/j.mechmat.2011.06.007.

[14] H. X. Li. Microscopic limit analysis of cohesive-frictional composites with nonassociated plastic flow. European Journal of Mechanics-A/Solids, 37, (2013), pp. 281-293. https://doi.org/10.1016/j.euromechsol.2012.07.005.

[15] H. X. Li. A microscopic nonlinear programming approach to shakedown analysis of cohesive-frictional composites. Composites Part B: Engineering, 50, (2013), pp. 32-43. https://doi.org/10.1016/j.compositesb.2013.01.018.

[16] C. V. Le, P. H. Nguyen, H. Askes, and D. C. Pham. A computational homogenization approach for limit analysis of heterogeneous materials. International Journal for Numerical Methods in Engineering, 112, (10), (2017), pp. 1381-1401. https://doi.org/10.1002/nme.5561.

[17] J.-S. Chen, C.-T. Wu, S. Yoon, and Y. You. A stabilized conforming nodal integration for Galerkin mesh-free methods. International Journal for Numerical Methods in Engineering, 50, (2), (2001), pp. 435-466.

[18] V. Carvelli, G. Maier, and A. Taliercio. Kinematic limit analysis of periodic heterogeneous media. CMES(Computer Modelling in Engineering \& Sciences), 1, (2), (2000), pp. 19-30.

[19] C. V. Le, M. Gilbert, and H. Askes. Limit analysis of plates using the EFG method and secondorder cone programming. International Journal for Numerical Methods in Engineering, 78, (13), (2009), pp. 1532-1552. https://doi.org/10.1002/nme.2535. 